\title{
Interaction of intense laser pulses with atomic clusters: Measurements of ion emission, simulations and applications
}

\author{
J.W.G. Tisch ${ }^{\text {a,* }}$, N. Hay ${ }^{\text {a }}$, K.J. Mendham ${ }^{\text {a }}$, E. Springate ${ }^{\text {a }}$, \\ D.R. Symes ${ }^{\text {a }, ~ A . J . ~ C o m l e y ~}{ }^{\text {a }}$, M.B. Mason ${ }^{\text {a }, ~ E . T . ~ G u m b r e l l ~}{ }^{\mathrm{a}}$, \\ T. Ditmire ${ }^{\text {b }, ~ R . A . ~ S m i t h ~}{ }^{\text {a }}$, J.P. Marangos ${ }^{\text {a }}$, M.H.R. Hutchinson ${ }^{\text {a }}$ \\ ${ }^{a}$ Blackett Laboratory, Imperial College, London $S W 72 B W, U K$ \\ ${ }^{\mathrm{b}}$ Department of Physics, University of Texas, Austin, TX 78712, USA
}

\begin{abstract}
This review paper provides a general introduction to the interaction of intense $\left(>10^{15} \mathrm{~W} \mathrm{~cm}^{-2}\right)$, femtosecond laser pulses with atomic clusters in the size range 500-105 atoms. A nanoplasma model of the laser-cluster interaction is used to elucidate the underlying physics. Measurements of ion emission from the laser-cluster interaction are presented together with numerical simulations. Emerging applications are described.
\end{abstract}

(c) 2003 Elsevier Science B.V. All rights reserved.

PACS: $36.40 . \mathrm{Gk} ; 52.50 . \mathrm{Jm}$

Keywords: Review; Laser; Cluster; Nanoplasma

\section{Introduction}

Physicists and chemists have been fascinated in atomic clusters since their discovery in the mid 1950s [1] because of the unique position clusters hold as an intermediate state between molecules and solids. While the optical properties of clusters have been studied for some time at low intensity, the advent of chirped pulse amplification (CPA) lasers [2] has allowed atomic clusters to be exposed to laser intensities in the range $10^{15}-10^{17} \mathrm{~W} \mathrm{~cm}^{-2}$ (laser pulse durations in the range $0.1-10 \mathrm{ps}$ ), where the electric field of the laser is greater than

${ }^{*}$ Corresponding author. Fax: +44-20-7594-7714.

E-mail address: john.tisch@ic.ac.uk (J.W.G. Tisch). atomic binding fields (the so-called non-perturbative regime) and where the wiggle energy of a free electron in the laser field (the ponderomotive potential) is in the range $0.1-10 \mathrm{keV}$ [3]. Following the first observation of "anomalous" X-ray lines from clustered gas targets in the early 1990s [4], many subsequent experimental studies (cited below) have shown that under intense, short pulse irradiation, clusters of size in the range $500-10^{6}$ atoms become a source of bright $\mathrm{X}$-rays, fast electrons (a few keV) and energetic, highly charged ions (up to $1 \mathrm{MeV}$ and $40^{+}$). This interaction is distinct from the Coulomb-explosion dominated dynamics observed in clusters at lower intensity, and much more energetic than that of the wellstudied cases of atoms [5-7], molecules [8,9] and small $(<\mathrm{a}$ few hundred atoms) clusters $[10,11]$ in 
intense laser fields. In terms of the efficient coupling of laser energy to electrons and ions, the laser-cluster interaction is more akin to the bulk solid interaction [12], i.e. displaying plasma-like behaviour. It should also be noted, that these nanometer scale clusters are considerably smaller than micron size droplets [13] and most microstructured targets that have been studied for some time [14].

In this paper, following a brief description of cluster formation, an introduction to the physics of the laser-cluster interaction will be provided, largely in the framework of the "nanoplasma model" [15]. This model treats the cluster in the strong laser field as a nanometer-scale spherical plasma, subject to the standard laser-plasma processes, such as inverse Bremsstrahlung heating, but taking into account the unique target geometry. Some illustrative examples of experimental results on ion emission from clusters will be summarised. Finally, some applications of the laser-cluster interaction will be presented, namely efficient debris-free X-ray generation and pulsed neutron production via nuclear fusion in deuterium clusters.

\section{Cluster formation in gas-jets}

Van der Waal bonded clusters can be formed in high pressure gas-jets (i.e. free-jet expansions) from the standard piezo or solenoid actuated pulsed valves used in pulsed laser experiments. Clustering occurs due to the cooling associated with the adiabatic expansion of the gas into vacuum. The cooling leads to a phase-transition and the coalescence of atoms into clusters. The properties of the resulting "cluster medium" are remarkable. It has the average density of a gas, but the individual, nanometer scale clusters are at near solid density.

\subsection{The Hagena parameter}

The onset of clustering and the size of clusters produced can be described by an empirical scaling parameter, $\Gamma^{*}$, known as the Hagena parameter [16]:
$\Gamma^{*}=k \frac{(d / \tan \alpha)^{0.85} p_{0}}{T_{0}^{2.29}}$,

where $d$ is the nozzle diameter (in $\mathrm{mm}$ ), $\alpha$ the expansion half-angle $\left(\alpha=45^{\circ}\right.$ for sonic nozzles, $\alpha<45^{\circ}$ for supersonic), $p_{0}$ the backing pressure (in mbar), $T_{0}$ the pre-expansion temperature (in Kelvin) and $k$, the condensation parameter, is a constant related to bond formation $\left(k_{\mathrm{He}}=3.85\right.$ while $k_{\mathrm{Xe}}=5500$ [17]). Gas-jets with the same $\Gamma^{*}$ tend to form clusters of the same average size. Cluster formation is a statistical process and therefore there is usually a relatively broad distribution of cluster sizes [17-19]. In the massive condensation regime, where nearly all the atoms have condensed into clusters of greater than $\sim 100$ atoms, the average number of atoms per cluster $\left\langle N_{\mathrm{c}}\right\rangle$ scales approximately as $[17,20]$

$\left\langle N_{\mathrm{c}}\right\rangle \sim \Gamma^{* 2.0}$.

By varying $T_{0}$ and $p_{0}$, it is possible to control the value of $\Gamma^{*}$ to engineer a cluster medium of arbitrary average density and cluster size. Optical Rayleigh scattering provides a convenient technique for the in situ measurement of average cluster sizes [21-23,78].

\subsection{Dense versus diffuse cluster targets}

Laser-cluster interactions fall into two broad categories. One involves the interaction with a very low density cluster medium and the other with a dense cluster medium. The former provides an approximation to the interaction of intense laser pulses with single clusters, which is important for understanding the physics without the complications introduced by macroscopic effects. The latter is required for applications where a high density of clusters is needed to produce a high energy-density plasma, or where macroscopic effects are essential to the process. The study of these two density regimes demands quite different experimental approaches. To allow the laser pulses to interact with a very low density of clusters, the laser is focused many hundreds of nozzle diameters below the nozzle into a cluster beam of density of order $10^{10} \mathrm{~cm}^{-3}$ that is produced with a skimmer and 
differential pumping arrangement. Interactions with dense cluster media are more straightforward. The laser is focused a few nozzle diameters beneath the nozzle of the gas jet, where the density of clusters can be as high as $5 \times 10^{16} \mathrm{~cm}^{-3}$.

\section{The nanoplasma model}

A variety of models of the laser-cluster interaction have been put forward. These include the inner shell excitation model (including coherent electron motion) $[4,24,25]$, the ionisation ignition model [26], Coulomb explosion models [27], particle codes [28,29] and nanoplasma models $[15,30,31]$. There is not space to review them all. Instead, the focus here will be on the nanoplasma model, developed by Ditmire et al. at Lawrence Livermore National Laboratory [15]. This numerical model has proved quite successful in reproducing most of the experimental results concerning ion (and electron) emission from "single" clusters [22] and provides an intuitive picture of the interaction in terms of the formation of a high-density, nanometer scale plasma (hence "nanoplasma"), ionisation and heating in the laser field and its subsequent rapid expansion ("explosion") leading to the formation of energetic electrons and ions. The impetus for the development of this model was experimental data demonstrating that large clusters in strong fields behave more like bulk solids than smaller clusters or large molecules, and appreciation of the fact that atomic clusters have near solid density, despite comprising a medium with the average density of a gas. This motivated a plasma description of the ionised cluster, despite its nanometer scale. A key aspect was the inclusion of shielding and enhancement of the laser field inside the cluster, which gives rise to a resonance in the collisional heating rate and provides an explanation for the very energetic nature of the interaction. This resonance leads to certain signature features in the ion emission from cluster explosions, such as an optimum cluster size and optimum laser pulse duration that maximise the ion kinetic energies. These signature features have been observed in experiments [32,33]. The existence of this resonance has also been verified experimentally in a study in which two pulses of different colours have been used to excite the resonance twice [34].

\subsection{Validity of plasma description}

A plasma treatment is only valid if the vast majority of the ionised electrons in the cluster are confined to the cluster during the laser pulse, to ensure quasi-neutrality. While for molecules and small clusters (less than a few hundred atoms) the electrons can escape, for large (thousands of atoms), high $Z$ clusters, the majority of the ionised electrons are confined by the space charge of the ions, as is typically the case for a solid. For low $Z$ clusters (e.g. H, D), however, it has been shown that most of the electrons can escape leading to highly charged clusters that rapidly Coulomb explode [35]. The second technical requirement for a plasma treatment is that the cluster size has to be much larger than the Debye length. For typical parameters (solid density Xe plasma, ionised to $5^{+}$ with an electron temperature of $1 \mathrm{keV}$ ) the Debye length is $\approx 0.5 \mathrm{~nm}$, much less than the $5-10 \mathrm{~nm}$ size of the clusters of interest. Finally, if the laser pulse is to interact with this nanoplasma then it must "hold together" on the time scale of the laser pulse (at least to the peak of the pulse). Assuming an expansion of the cluster at the plasma sound speed, the time taken for it to expand to a background gas density of $\approx 10^{17} \mathrm{~cm}^{-3}$ is typically around $1 \mathrm{ps}$ (for the same parameters as before). Clearly, intense laser pulses of femtosecond duration are required.

\subsection{Simplifying assumptions}

The model assumes a spherical plasma with all its constituent particles simultaneously experiencing the same (time-dependent) laser electric field strength. This is valid since the clusters considered $\left(<10^{6}\right.$ atoms, $<10 \mathrm{~nm}$ in diameter) are much smaller than both the laser wavelength $(\approx 800 \mathrm{~nm})$ and the plasma skin depth. It is also assumed that the temperature distribution across the plasma is isotropic, since the plasma is small enough and collisional enough to prohibit temperature gradients. 
Further, the ion density distribution is assumed to be uniform across the cluster and the expansion is assumed to be self-similar, so the density remains uniform across the cluster throughout its expansion. The final important assumption is that the electron energy distribution is Maxwellian at all times, which will be true in the limit of extremely rapid thermalisation.

\subsection{Cluster ionisation}

Ionisation of the cluster begins with laser fieldionisation on the rising edge of the laser pulse that liberates a small number of electrons, with collisional ionisation then becoming important. Field ionisation is described in the model by ADK tunnel ionisation rates [36] and collisional ionisation using the empirical formula of Lotz [37]. Both thermal and laser-driven collisional ionisation is accounted for. In fact, collisional ionisation is by far the dominant ionisation mechanism in the nanoplasma owing to its high density, leading to the production of highly charged ions. For example, the Lotz collisional ionisation rate for typical nanoplasma conditions (Ar plasma ionised to $8^{+}$, electron density of $10^{23} \mathrm{~cm}^{-3}$ ) can be $0.1 \mathrm{fs}^{-1}$ or higher for typical thermal and quiver electron energies of around a few hundred $\mathrm{eV}$. At this rate, $100 \%$ ionisation to $\mathrm{Ar}^{9+}$ occurs in 10 fs or less, highlighting the extremely fast timescales involved.

\subsection{Cluster heating}

The nanoplasma model provides an explanation for the much higher ion energies seen from large clusters compared to molecules and small clusters in terms of highly efficient collisional heating (inverse Bremsstrahlung) in the high density nanoplasma, as is seen in solid target laser plasmas. Above threshold ionisation heating [38] is also included, but its contribution is small $(<100 \mathrm{eV})$. The collisional heating of the cluster is calculated in the model as the heating of a uniform dielectric sphere in the time-varying electric field of the laser. Inside the dielectric sphere, the electric field is [39]

$E=\frac{3 E_{0}}{|\varepsilon+2|}$, where $E_{0}$ is the external field and the plasma dielectric constant is given by a Drude model

$\varepsilon=1-\frac{n_{\mathrm{e}} / n_{\text {crit }}}{1+i v / \omega}$.

Here $n_{\mathrm{e}}, n_{\text {crit }}$ are the electron and critical plasma densities, $\omega$ is the laser frequency and $v$ the electron-ion collision frequency that is calculated from the Silin formulae [40]. When $n_{\mathrm{e}}=3 n_{\text {crit }}$, $|\varepsilon+2|$ goes through a minimum and the field inside the cluster is greater than the external field. At this resonance, the cluster heating rate is also increased, even though the electron-ion collision frequency is actually reduced. Another consequence of the geometry of the laser-cluster interaction is that there is no heat conduction possible to surrounding "cold" material, as is the case in a bulk plasma, suggesting that very high plasma temperatures may be attained in the nanoplasma.

\subsection{Cluster expansion}

The expansion of the nanoplasma is driven by two pressures, the Coulomb pressure arising from repulsion between ions following a charge build-up Qe on the cluster of radius $r$ :

$P_{\text {Coul }}=\frac{Q^{2} e^{2}}{8 \pi r^{4}}$,

and the hydrodynamic pressure from the hot electrons (one can think of the hot electrons expand outwards, dragging the ions with them):

$P_{\mathrm{H}}=n_{\mathrm{e}} k T_{\mathrm{e}}$,

where $k$ is the Boltzmann constant and $T_{\mathrm{e}}$ the electron temperature. The $Q / r^{4}$ scaling of $P_{\text {Coul }}$ shows that it will be important for small clusters or for low $Z$ clusters where the electrons are not confined and $Q$ can become large. The hydrodynamic pressure scales as $r^{-3}$ (since $n_{\mathrm{e}} \sim$ volume ${ }^{-1}$ ), so is therefore more important for larger clusters. The internal pressure driving the cluster apart can be huge. For realistic nanoplasma conditions $\left(n_{\mathrm{e}}=10^{23} \mathrm{~cm}^{-3}, k T_{\mathrm{e}}=1 \mathrm{keV}\right)$, the hydrodynamic pressure, $P_{\mathrm{H}} \approx 100$ Mbar. It is hardly surprising that the end result is an explosion of the nanoplasma that gives rise to a shrapnel of high energy ions and electrons. The cluster expansion rate is 
calculated in the model by equating the rate of change of the cluster kinetic energy (proportional to the total pressure $\left.P_{\text {Coul }}+P_{\mathrm{H}}\right)$ with the rate at which work is done by the plasma in its expansion [41]. At any stage in the interaction the model allows the relative significance of the Coulomb and hydrodynamic pressures to be compared. Simulations shows that $P_{\mathrm{H}}$ dominates over $P_{\text {Coul }}$ for $\mathrm{Ar}$, $\mathrm{Kr}$ and $\mathrm{Xe}$ clusters greater than $r>2 \mathrm{~nm}$.

\subsection{Cluster charge build-up}

The charge build-up on the cluster is due to electrons free-streaming from the surface of the nanoplasma. The model allows an electron to freestream if it is less than one mean free path (in the direction of travel) from the surface and if its energy is greater than the escape energy of the cluster. The mean free path is calculated using the Spitzer formula [42]. The escape energy is calculated from the potential energy on the surface of a sphere with charge $Q$, assuming that the charge is distributed isotropically over the sphere and scales as $Q / r$. Free-streaming of the most energetic electrons depletes the hot tail of the Maxwellian electron distribution. Electron emission data [43] suggest that the rethermalisation - though extremely rapid - is not always fast enough to completely repopulate this tail. However, the assumption of a Maxwellian electron energy distribution is overall a good one.

\subsection{Ion energies from cluster explosions}

Electron-ion collisions heat up the electrons efficiently, but not the ions. This can be seen from the electron-ion equilibration time. For typical nanoplasma conditions and for initially cold ions, it is in excess of $30 \mathrm{ps}$ - much longer than the nanoplasma disassembly time. In other words, there is insufficient time for the electron energy to be transferred to the ions through collisions. Instead, the ions gain energy in the hydrodynamic expansion (where the thermal energy of the electrons is converted to radial motion ion kinetic energy) and/or through Coulomb explosion. For a hydrodynamic expansion at the plasma sound speed, the mean ion energy will be of order $\left\langle E_{\text {ion }}\right\rangle \approx\langle Z\rangle k T e$,

where $\langle Z\rangle$ is the mean ion charge state. This can be on the order of $40-50 \mathrm{keV}$ for multi-keV electron temperatures and highly charged ions $(\langle Z\rangle \approx 20-$ see Section 4.3), consistent with the mean ion energies observed [22]. Coulomb explosion is the main process by which ions gain kinetic energy for molecules and small clusters in intense laser fields [9]. This is important for large, low $Z$ clusters where charge build-up is substantial. For high $Z$ clusters, it provides an explanation for the fastest ions observed [44].

\subsection{Numerical simulation of a cluster explosion}

The calculated time-history of a cluster of 5000 Xe atoms irradiated by a $200-\mathrm{fs}, 780-\mathrm{nm}$ laser pulse with a peak intensity of $10^{16} \mathrm{~W} \mathrm{~cm}^{-2}$ is illustrated in Fig. 1. The time-dependence of the nanoplasma parameters clearly shows field enhancement in the cluster, a resonance in the heating and an extremely rapid expansion (explosion) of the nanoplasma. The peak of the laser pulse is at $t=0 \mathrm{fs}$. At around $t=-280 \mathrm{fs}$, when the intensity is $\sim 4 \times 10^{13} \mathrm{~W} \mathrm{~cm}^{-2}$, a small number of free electrons are created through tunnel ionisation. The electron density rises to reach $3 n_{\text {crit }}$ at $t=-270 \mathrm{fs}$ (Fig. 1(c)). At this point the field in the cluster is enhanced (Fig. 1(a)) and more electrons are liberated through tunnel, laser-driven and thermal ionisation. The electron density is now higher than $3 n_{\text {crit }}$ and the field inside the cluster is shielded from the external laser field. The tunnel and laser-driven ionisation rates fall off, but electrons are still created through thermal collisions.

From $t=-50 \mathrm{fs}$ onwards, some electrons are able to leave the cluster, as the mean electron temperature is in the region of $100-1000 \mathrm{eV}$ and the escape energy is $\sim 200-2000 \mathrm{eV}$. The combined effect of the free-streaming of electrons out of the cluster and the hydrodynamic expansion of the cluster is that the electron density starts to fall, after peaking at over $50 n_{\text {crit }}$. The field in the cluster again starts to rise as the electron density drops, so the tunnel and laser-driven ionisation rates increase while the thermal collisional ionisation rate falls. Near the peak of the laser pulse, at $t=-12$ 

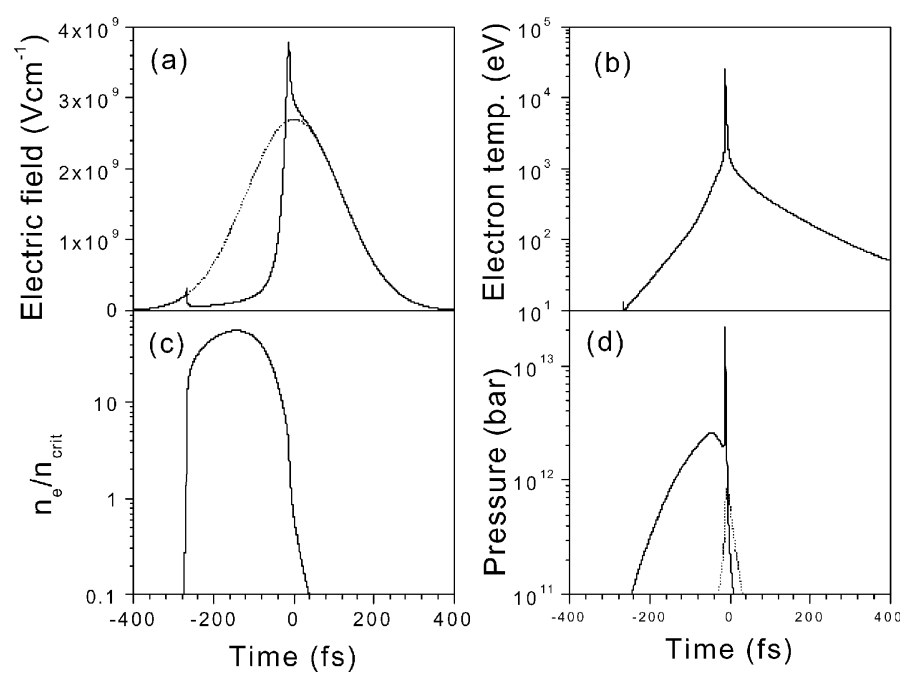

Fig. 1. Calculated time history of the explosion of a 5000 -atom Xe cluster irradiated by a $200 \mathrm{fs}, 780 \mathrm{~nm}$ laser pulse with a peak intensity of $1 \times 10^{16} \mathrm{~W} \mathrm{~cm}^{-2}$. (a) Envelope of the laser pulse (dotted line) and the field inside the cluster (solid line). (b) Electron temperature inside the cluster. (c) Electron density/critical electron density. (d) Hydrodynamic (solid line) and Coulomb (dotted line) pressure.

fs, the electron density in the cluster drops to $3 n_{\text {crit }}$. The resonantly increased heating rate causes the electron temperature in the cluster to soar to 25 $\mathrm{keV}$ (Fig. 1(b)). The field in the cluster is also strongly enhanced and the peak intensity in the cluster reaches $2 \times 10^{16} \mathrm{~W} \mathrm{~cm}^{-2}$, twice the intensity outside. The electron free-streaming rate increases sharply as a significant number of electrons have energies above the then 4-keV escape energy.

The total charge on the cluster increases to $5.5 \times 10^{4} e$, resulting in the Coulomb pressure increasing to 10 Mbar (Fig. 1(d)). However, this is small compared to the hydrodynamic pressure due to the hot electrons of $200 \mathrm{Mbar}$. This pressure causes a sharp increase in the cluster expansion velocity. This is the explosion of the cluster. Once the nanoplasma density has dropped to $\approx 10^{17}$ $\mathrm{cm}^{-3}$, the final expansion velocity of electrons and ions is $3.3 \times 10^{7} \mathrm{~cm} \mathrm{~s}^{-1}$, which corresponds to a maximum ion energy of $\sim 80 \mathrm{keV}$. The final electron energy is much lower, only $30 \mathrm{eV}$, a consequence of their much lighter mass. However, electrons that free streamed away from the nanoplasma have energies in the $0.2-2 \mathrm{keV}$ range.

This simulation shows an extremely energetic laser-cluster interaction, with ion energies close to
$100 \mathrm{keV}$ and electron energies of several $\mathrm{keV}$. We will see that these predictions are borne out in experiments.

\section{Experimental measurements of ion emission}

The first evidence of highly charged ion production from intense laser-cluster interactions was the observation of "anomalous" X-ray emission lines corresponding to highly stripped ions [4]. Strong M- and L-shell emission was observed from $\mathrm{Kr}$ and $\mathrm{Xe}$ clusters targets, including a component attributed to $\mathrm{Kr}^{9+}$. Shortly afterwards, high charge states of I and Ar ions were seen from small $\mathrm{HI}$ and $\mathrm{HIAr}_{n}$ clusters exposed to intense, femtosecond pulses [45]. For these small clusters a Coulomb explosion model could explain the ion energies that were a few hundred $\mathrm{eV}$. This was followed by the observation at Imperial College of $\mathrm{keV}$ electrons emitted from large Xe clusters irradiated by femtosecond pulses of intensity $\sim 10^{16} \mathrm{~W} \mathrm{~cm}^{-2}$, suggestive of a solid target laser-plasma interaction [43]. In the same issue of Physical Review Letters, observation of very highly charged ions from $\mathrm{Xe}$ and $\mathrm{Kr}$ clusters was reported [46] - using 
femtosecond pulses at an intensity in the vicinity of $10^{15} \mathrm{~W} \mathrm{~cm}^{-2}, \mathrm{Xe}^{20+}$ and $\mathrm{Xe}^{18+}$ were seen in ion time-of-flight (TOF) spectra. Even higher charge states (up to $\mathrm{Xe}^{40+}$ ) were then seen by the Imperial College physicists, who also observed average ion energies of $\sim 45 \mathrm{keV}$ with a maximum extending to $1 \mathrm{MeV}$ in the interaction of femtosecond laser pulses (again $\sim 10^{16} \mathrm{~W} \mathrm{~cm}^{-2}$ ) with several thousand atom rare-gas clusters $[47,48]$. The transition from molecular to plasma behaviour was now manifest. These first direct measurements of high energy, highly charged ions were later confirmed and extended by other groups $[44,49]$. In the following sections we summarise the ion emission data obtained at Imperial College. Ahead of this, a short description of the Imperial College laser system and interaction chamber is given.

\subsection{Experimental apparatus}

\subsubsection{Laser system}

A femtosecond, CPA laser system based on titanium doped sapphire (Ti:S) provided pulses for the Imperial College cluster work, centred at a wavelength of $780 \mathrm{~nm}$, with energy up to $60 \mathrm{~mJ}$ and a pulse duration of $<200$ fs [50]. The frontend of the system is a Kerr lens mode-locked Ti:S oscillator that generates near transform-limited pulses of duration $\sim 90$ fs. The oscillator pulses are stretched in a diffraction-grating stretcher to a duration of $\sim 250 \mathrm{ps}$, thus reducing the pulse power by a factor $\sim 3000$. This permits safe amplification first to the millijoule level in a Ti:S regenerative amplifier operating at $10 \mathrm{~Hz}$ and then to $\sim 120 \mathrm{~mJ}$ in a multi-pass Ti:S power amplifier. The amplified pulses are then recompressed in a grating compressor to yield pulses at a power level of approximately $0.5 \mathrm{TW}(60 \mathrm{~mJ} /$ $150 \mathrm{fs})$ at a pulse repetition rate of $10 \mathrm{~Hz}$. In conjunction with standard (single-shot) autocorrelation pulse duration measurements, and equivalent-plane focal spot characterisations, the focused laser intensity is inferred from ion appearance data using over-the-barrier ionisation (OTBI) thresholds [51]. This is carried out in the same interaction chamber (described below) used for the low-density cluster studies, providing in situ intensity measurement.

\subsubsection{Interaction chamber}

The interaction chamber, illustrated in Fig. 2, is essentially a TOF particle spectrometer coupled to a skimmed, gas-jet cluster source. It is used to measure the energies of electrons and ions as well as the charge states of ions produced in the laser induced explosion of isolated clusters. As outlined above, to achieve a relatively low density of clusters in the interaction region, a cluster jet produced from a solenoid pulsed-valve at the top of the chamber is skimmed to produce a much lower density cluster beam. The cluster beam intersects the laser focus at the centre of the high vacuum section of the chamber, some $50 \mathrm{~cm}$ below the nozzle. Differential pumping is employed to maintain the high vacuum in the main chamber. Typically, the density of clusters at the laser focus is $\sim 10^{10} \mathrm{~cm}^{-3}$ corresponding to $<1000$ clusters in the laser focal volume.

The linearly polarised laser is focused at $\sim f / 20$ with plano-convex lens which gives an approximately Gaussian focal spot of $\sim 20 \mu \mathrm{m}$ ( $e^{-2}$ radius). By rotating a half-wave plate before the lens, the polarisation vector can be rotated through $360^{\circ}$, enabling the angular distribution of particles in the plane perpendicular to the laser axis to be studied.

The energies of ions produced in the interaction are determined from their TOF from the laser focus to a microchannel plate (MCP) detector, without the use of an extraction field. The electrons produced in cluster explosions are too fast for standard TOF techniques to be used. Instead, a retardation method is employed. Two closely spaced grids are placed in the flight path. To the first, a voltage $\Phi$ is applied, the second is grounded. This introduces a potential barrier to electrons with energy less than $e \Phi$, where $e$ is the electron charge. The MCP signal is recorded as a function of $\Phi$. The electron energy spectrum is then found by differentiating this distribution with respect to $\Phi$.

With a small modification to the standard TOF apparatus, information can also be gained on the ion charge state, $Z$. Three closely spaced metal grids are placed in the flight tube. Applying a potential $\Phi$ to the middle grid, while keeping the front and back grids at earth introduces a barrier 


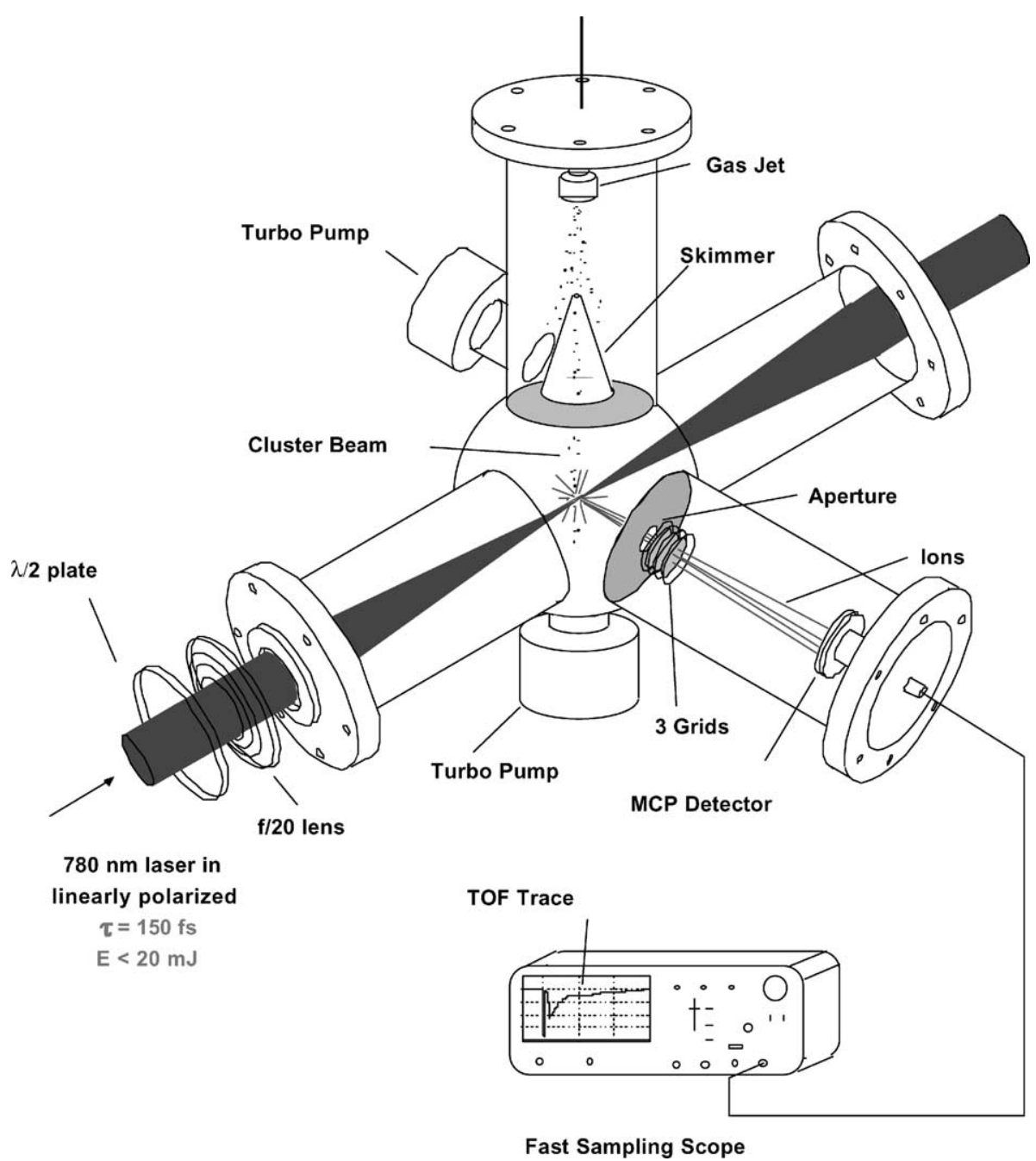

Fig. 2. TOF spectrometer used to measure electron and ion energies and ion charge states from exploding clusters in the Imperial College experiments.

to ions with energy less than $Z e \Phi$, without significantly altering the flight time of higher energy ions. By measuring the number of ions reaching the detector as a function of $\Phi$, and then differentiating with respect to $\Phi$, the charge state distribution of the ions as a function of their kinetic energy can be determined. However, this technique cannot measure the energy distribution of a given charge state. The complete charge state information can be gained by incorporating magnetic deflection into the ion flight path, as demonstrated in [49].

\subsection{Ion energy spectrum}

Typical raw ion TOF spectra are shown in Fig. 3(a) for a range of angles between the laser polarisation and the TOF axis. Each spectrum is averaged over several thousand laser shots. The target was 2500-atom Xe clusters irradiated at $2 \times 10^{16} \mathrm{~W} \mathrm{~cm}^{-2}$. The fast unresolved feature in the TOF spectrum is due to the emission of energetic electrons (resolved in other studies [43]), the broader peak is due to the ions. The close similarity of the TOF spectra recorded for different 

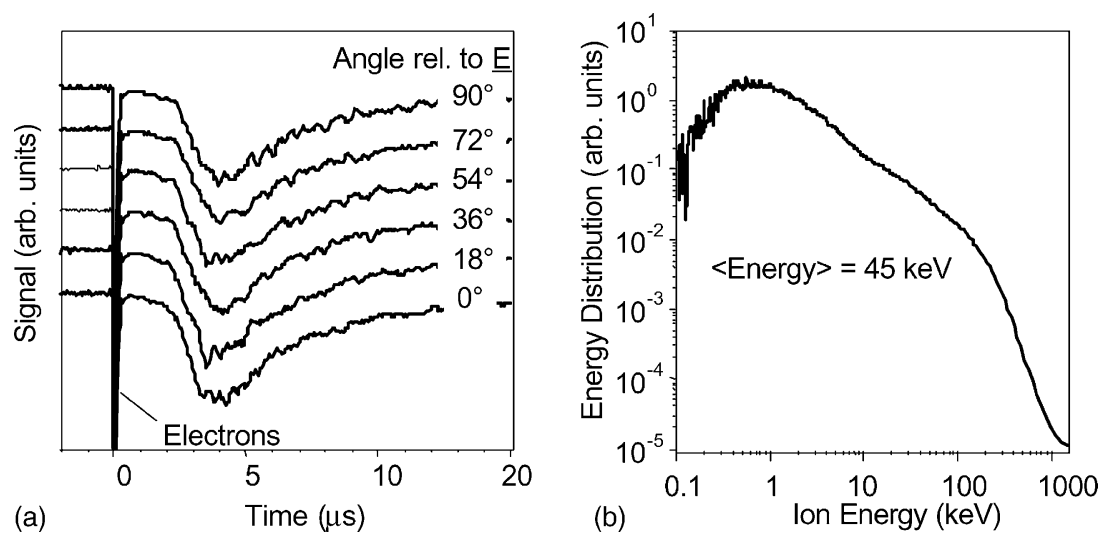

Fig. 3. (a) Raw ion TOF spectra from the explosion of 2500 -atom Xe clusters irradiated at $2 \times 10^{16} \mathrm{~W} \mathrm{~cm}^{-2}$ for different angles between the laser polarisation and TOF axis (the flight distance was $0.38 \mathrm{~m}$ ). (b) Energy spectrum corresponding to 0 curve. The mean energy is $45 \mathrm{keV}$ and a fraction of the ions have energies greater than $1 \mathrm{MeV}$.

angles is a clear signature of a spherically symmetric explosion. The energy spectrum corresponding to the $0^{\circ}$ trace is displayed in Fig. 3(b) (the other angles give almost identical energy spectra). The mean ion energy is $45 \pm 5 \mathrm{keV}$, showing that the average laser energy deposited per ion is substantial. A remarkable aspect of the ion spectrum is the presence of ions with energies up to $1 \mathrm{MeV}$. This energy is four orders of magnitude higher than has previously been observed in the Coulomb explosion of molecules [52] and about 1000 times higher than the average energy of the highest charge state $\mathrm{Ar}$ ions ejected in the disintegration of small ( $<10$ atoms) cluster. In fact $1 \mathrm{MeV}$ is about a factor four higher than the predictions of the nanoplasma model for these parameter values. It has been suggested that the $\mathrm{MeV}$ ions do not result from the hydrodynamic expansion, but are the result of an electrostatic shock wave that Coulomb ejects the most highly charged ion that are close to the cluster surface [44].

\subsection{Ion charge state distributions}

The production of highly stripped ions is another characteristic feature of the laser-cluster interaction. The charge state distributions of Xe ions (for the same conditions as Fig. 3) are shown in Fig. 4 as a function of their kinetic energy. For the

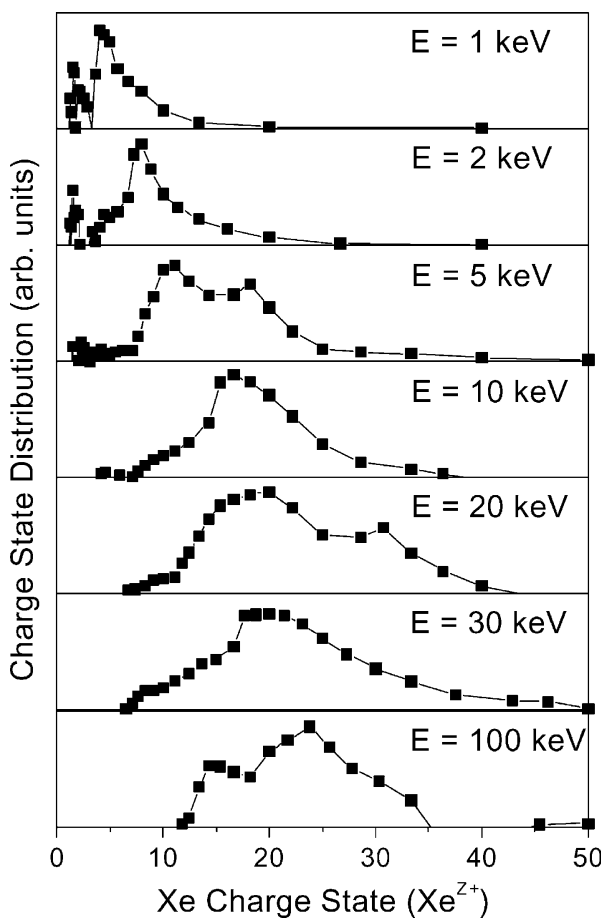

Fig. 4. Measured charge-state distributions from the explosion of 2500-atom Xe clusters irradiated at $2 \times 10^{16} \mathrm{~W} \mathrm{~cm}^{-2}$ for ion kinetic energies of $1,2,5,10,20,30$ and $100 \mathrm{keV}$.

high energy ions $(>100 \mathrm{keV})$, the peak charge state is at $Z=18^{+}-25^{+}$, with some ions, remarkably, having charge states as high as $40^{+}$[47]. These are much higher than the $\sim 12^{+}$expected from laser 
field ionisation of single atoms at these intensities according to an OTBI model [53]. In fact, the OTBI model predicts an intensity of nearly $10^{20}$ $\mathrm{W} \mathrm{cm}{ }^{-2}$ to produce such a high charge state. The nanoplasma model provides an explanation for these high charge states. Efficient collisional heating in the nanoplasma heats the electrons to several $\mathrm{keV}$. These electrons strip the ions to high charge states through collisions. Modelling shows that it is largely thermal collisional ionisation responsible, which occurs while the cluster is shielded from the external field. The ion charge state depends only weakly on ion kinetic energy, contrary to what would be expected from a pure Coulomb explosion. Using a magnetic deflection TOF spectrometer, the energy distributions of ion charge states in the range $1-30^{+}$from 2 millionatom Xe clusters interacting with 130-fs Ti:S pulses at an intensity of $\sim 5 \times 10^{17} \mathrm{~W} \mathrm{~cm}^{-2}$ have been measured [44]. This allowed the dependence of the ion energies upon the charge states to be investigated.

\subsection{Parameter scalings}

In this section we look at how the ion energies from cluster explosions scale with two important experimental parameters, the cluster size and the laser intensity [54]. These scaling provide tests of the models and help identify the conditions that maximise the ion energies, which is important for applications.

\subsubsection{Cluster size}

Fig. 5 shows how the maximum ion energy varies with the number of atoms in a Xe cluster in the range 200-74,000 Xe atoms [54]. A laser intensity of $3 \times 10^{15} \mathrm{~W} \mathrm{~cm}^{-2}$ was used. The threshold size for energetic ion production $(>1 \mathrm{keV})$ appears to be $\sim 200-400$ atoms. The maximum ion energy rises from $8 \mathrm{keV}$ at 200 atoms/cluster to a peak of $\sim 53 \mathrm{keV}$ for clusters of $\sim 8000$ atoms before falling to $\sim 28 \mathrm{keV}$ as the cluster size is increased beyond 50,000 atoms/cluster. $\mathrm{Kr}$ and Ar clusters show a qualitatively similar behaviour.

The solid curve is a numerical simulation from the nanoplasma model, which is in reasonable agreement with the experimental data, though the

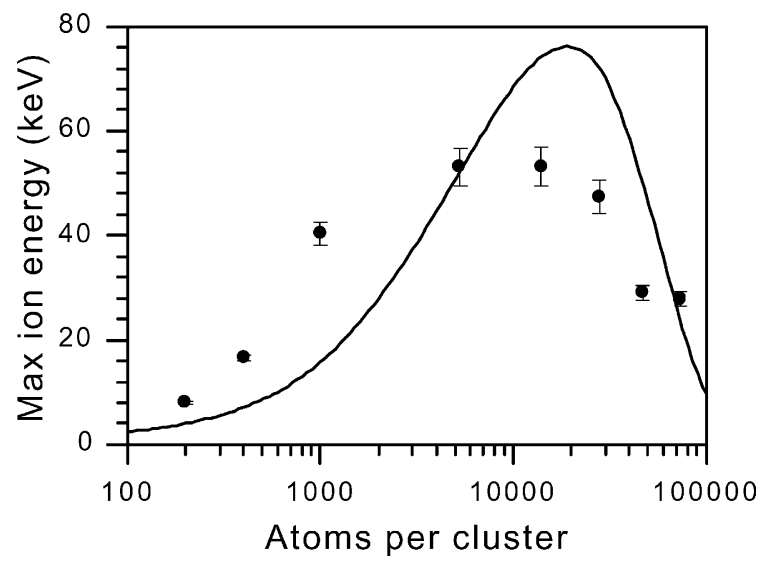

Fig. 5. Measured (solid points) and calculated (line) maximum ion energies as a function of cluster size for Xe clusters interacting with $230 \mathrm{fs}, 780 \mathrm{~nm}$ pulses at a peak intensity of $3 \times 10^{15}$ $\mathrm{W} \mathrm{cm}{ }^{-2}$.

calculated optimum size is somewhat larger. However, there is a factor of two uncertainty in the cluster size measurements and further, the calculation does not take into account the distribution of cluster sizes present in the laser focus. The modelling has recently been improved to include a realistic cluster size distribution [55].

The existence of an optimum cluster size is strong evidence for the $n_{\mathrm{e}}=3 n_{\text {crit }}$ resonance predicted by the nanoplasma model. The maximum ion energy is determined largely by the laser intensity when the cluster experiences the resonant heating. The highest ion temperatures are obtained when the cluster passes through the $n_{\mathrm{e}}=3 n_{\text {crit }}$ point near the peak of the laser pulse. Small clusters expand too quickly and reach this point before the peak. Larger clusters pass through $3 n_{\text {crit }}$ well after the peak intensity is reached. This also explains the existence of an optimum laser pulsewidth for a given cluster size seen in $[33,56]$.

An empirical formula for the optimum cluster size has been determined from the results of numerous simulations using the nanoplasma model [57]:

$N_{\mathrm{c}}$ (opt) $=1.8 \times 10^{-13} Z^{-1} I^{0.75} \tau^{3}$,

where $N_{\mathrm{c}}$ is the number of atoms in the cluster, $Z$ is the atomic number, $I$ is the laser intensity in $\mathrm{W} \mathrm{cm}{ }^{-2}$ and $\tau$ is the laser pulse duration in fs. 


\subsubsection{Laser intensity}

Fig. 6 shows how the maximum ion energies vary with the peak laser intensity $(230 \mathrm{fs}, 780 \mathrm{~nm}$ pulses) for 5300-atom Xe clusters and 6200-atom $\mathrm{Kr}$ clusters [54]. In Xe, a sharp onset of fast ion production is seen at $6 \times 10^{14} \mathrm{~W} \mathrm{~cm}^{-2}$. The ion energies rise steeply up to $\sim 10^{15} \mathrm{~W} \mathrm{~cm}^{-2}$, to an energy of $\sim 50 \mathrm{keV}$. Above $10^{15} \mathrm{~W} \mathrm{~cm}^{-2}$, the ion energies saturate, increasing as $\sim I^{0.2}$ (the integrated ion yield scale as $\sim I^{1.4}$, consistent with the increase in focal volume). At $10^{16} \mathrm{~W} \mathrm{~cm}^{-2}$, the maximum ion energy is $90 \mathrm{keV}$. The $\mathrm{Kr}$ ion energies follow a similar trend to the $\mathrm{Xe}$, with a sharp increase up to a mean energy of $9 \mathrm{keV}$ at $10^{15}$ $\mathrm{W} \mathrm{cm}{ }^{-2}$, followed by a slow increase up to energies of $75 \mathrm{keV}$ at $10^{16} \mathrm{~W} \mathrm{~cm}^{-2}$. For $\mathrm{Kr}$ the ion energies are about 20\% lower. The solid and dotted curves are the nanoplasma calculations for $\mathrm{Xe}$ and $\mathrm{Kr}$, respectively. Again, the agreement is reasonable.

The modelling provides explanations for the well-defined "appearance intensity" for energetic ion production, as well as the eventual saturation in the ion energies with increasing intensity. Referring back to Fig. 1(c), we see that the energetic cluster explosion is triggered by the second occurrence of the $n_{\mathrm{e}}=3 n_{\text {crit }}$ resonance, since the first one occurs very early in the laser pulse when the intensity is low. Modelling shows that the ap-

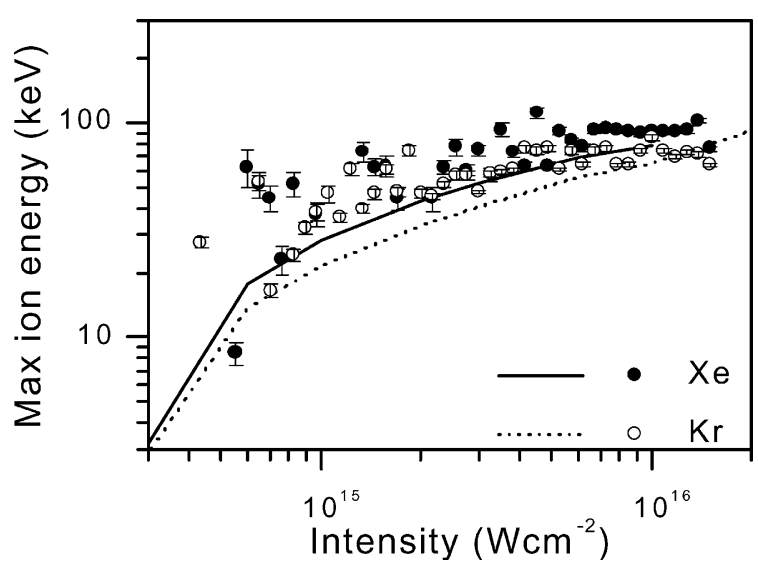

Fig. 6. Maximum ion energies measured as a function of laser intensity for 5300-atom Xe clusters (solid points) and 6200atom $\mathrm{Kr}$ clusters (open points), together with the simulated scaling for Xe clusters (solid line) and $\mathrm{Kr}$ clusters (dotted line). pearance intensity is just the minimum peak intensity required to ensure two occurrences of the resonance during the laser pulse. Below this intensity, the resonance is achieved only once, early in the laser pulse, because the expansion velocity is insufficient compared to the ionisation rate to drop $n_{\mathrm{e}}$ back down to $3 n_{\text {crit }}$. The saturation in the ion energies with increasing intensity is a product of the increased ionisation that occurs on the rising edge of the pulse. This results in the cluster expanding faster and reaching the second resonance earlier in the pulse. It therefore does not experience a substantially higher laser intensity at the point of resonant heating.

\section{Applications}

So far, we have concentrated on the explosions of individual clusters that have been studied in experiments with low density cluster beams and described theoretically in terms of the nanoplasma model. Now we turn to applications which are carried out in extended (i.e. typically a cylinder of length $\sim 1 \mathrm{~mm}$ as set by the gas jet length and diameter around $50 \mu \mathrm{m}$ corresponding to the laser focal spot size), high density cluster media (see Section 2.2) where the density of clusters is $\sim 10^{6}$ times greater. Extremely high laser absorption (up to $90 \%$ ) has been observed in such targets [58] - a remarkable result considering the target has the average density of a gas (at about atmospheric pressure) and is completely transparent to low intensity light. The deposition of almost all the laser pulse energy (a large fraction of a Joule in [58]) into a focal volume of typically $\sim 5 \times 10^{-6} \mathrm{~cm}^{-3}$ leads to the production of a very high energy density plasma that emits copious quantities of $\mathrm{X}$-rays in the $0.1-10 \mathrm{keV}$ range and can provide an environment for nuclear fusion, as we see below.

\subsection{X-ray generation}

$\mathrm{X}$-ray generation is currently the most important application of the laser-cluster interaction. The main attractions of this source, particularly 
for photolithographic applications, are that it produces very little debris, in contrast to solid targets (which is very important since expensive $\mathrm{X}$-ray collection optics must be positioned close to the target), and that it can be operated at a very high repetition rate. In fact, so attractive is the source that several large microchip manufactures are funding research to investigate it as an XUV photolithographic source for microchip production [59]. On a less commercial note, the prompt (likely to be $<1$ ps in duration) X-rays emitted by the cluster nanoplasmas immediately after heating by the laser allow the investigation of high-density plasmas through spectroscopic means [60], in the absence of significant absorption and re-emission of radiation that plagues such measurement of solid target laser plasmas.

In addition to a prompt X-ray component, time resolution of the X-ray emission using an X-ray streak-camera has shown that the most of the emission (around 99\%) is emitted on a nanosecond timescale [15,61]. This implies that it is coming from the hot, underdense $\left(n_{\mathrm{e}}<n_{\text {crit }}\right)$ plasma that is formed after the individual clusters have exploded and expanded to form a uniform, bulk plasma. The plasma emission is dominated by line emission from the resonance lines of highly charged ions in the plasma, which are populated initially by collisions with hot electrons, and then, after the plasma has cooled, via three body recombination [15]. Conversion of up to $10 \%$ of the incident laser energy into X-rays in the $17-30 \mathrm{~nm}$ range (e.g. $4 \mathrm{p}-3 \mathrm{~d}$ in $\mathrm{Kr}^{10+}$ ) in Ar clusters was reported in [15] using $30 \mathrm{~mJ}, 130 \mathrm{fs}$ laser pulses. This is comparable with the yields from solid targets. Many experiments have studied the yields and parameter scaling of the X-ray emission with a view to applications [62,56,63-65]. This continues to be an active area of research. One area of controversy is the scaling of the X-ray signal with the laser wavelength, with some groups claiming a very strong scaling, favouring shorter wavelength lasers such as $\mathrm{KrF}$ lasers at $248 \mathrm{~nm}$ [66], while others finding evidence of a much weaker scaling [67,79]. However, no systematic study has been carried out over a wide range of wavelengths ensuring identical focusing conditions and using exactly the same cluster source.

\subsection{Nuclear fusion}

The energetic laser-cluster interaction has opened the door to nuclear fusion experiments using table-top laser systems operating at high repetition rates. The $\mathrm{D}+\mathrm{T} \rightarrow n+\mathrm{He}^{4}$ reaction has by far the largest cross-section for the range of ion energies accessible in laser-cluster experiments, but tritium poses a serious radiological hazard and so the D-D reaction has been investigated instead. Half the D-D fusion events follow the $\mathrm{D}+\mathrm{D} \rightarrow$ $n(2.45 \mathrm{MeV})+{ }^{3} \mathrm{He}$ reaction, which is convenient for study since the neutron can escape the interaction chamber and, being of well-defined energy, can be identified unambiguously through TOF measurements.

The first definitive observation of a cluster based fusion reaction was reported in 1999 [68]. A dense $\mathrm{D}_{2}$ cluster target was irradiated with a $35 \mathrm{fs}, 200 \mathrm{~mJ}$ laser pulse from a Ti:S CPA system. In the target, deuterons ejected from different clusters collide with sufficient energy (multi-keV) to trigger fusion events. The low value of the condensation parameter, $k$, for $\mathrm{D}_{2}$ requires it to be cooled below $80 \mathrm{~K}$ to allow large cluster formation. Around 50,000 neutrons were observed per shot (at a repetition rate of $10 \mathrm{~Hz}$ ), which is a relatively large number when normalised to the input laser energy. In contrast to high $Z$ clusters (Ar, $\mathrm{Kr}, \mathrm{Xe}$ ), $\mathrm{D}_{2}$ clusters can be stripped of almost all their electrons by a sufficiently short laser pulse ( $<50 \mathrm{fs}$ is required). Hence a Coulomb explosion picture, rather than a nanoplasma model is appropriate. For a fully stripped $\mathrm{D}_{2}$ cluster of radius $r$, the maximum ion energy from the Coulomb explosion is given by

$E_{\max }=\frac{2 n_{\mathrm{D}} e^{2} r^{2}}{3 \varepsilon_{0}}$

where $n_{\mathrm{D}}$ is the initial ion density $\left(\sim 3 \times 10^{22} \mathrm{~cm}^{-3}\right.$ for $\mathrm{D}_{2}$ ). From this it can be calculated that a $\mathrm{D}_{2}$ cluster of radius $r>2.5 \mathrm{~nm}$ is required to produce multi-keV deuterons necessary for fusion. A detailed description of this work can be found in [69].

Modelling of the neutron yield within this Coulomb explosion picture has been carried out [70] providing good agreement with the experimental data, and a comparison with the case of a hydrodynamic cluster expansion is given in [71]. 
Also, direct measurements of proton energies up to $7 \mathrm{keV}$ from exploding hydrogen clusters have been made [72], showing a combination of Coulomb explosion and hydrodynamic mechanisms for somewhat longer laser pulses (90 fs).

Fusion in clusters has attracted considerable interest because it provides a pulsed source of neutrons of a small source size. The neutron pulse duration is basically set by the time taken for an energetic deuteron to leave the laser focus, and has been measured to be less than 500 ps [73], which is considerably shorter than $Z$-pinches and spallation devices. A number of schemes have been proposed to increase the number of neutrons per laser shot. The use of mixed species (or heteronuclear) clusters comprising deuterium and a higher $Z$ atomic species (e.g. DI, $\mathrm{D}_{2} 0, \mathrm{CD}_{4}$ ) is likely to increase the deuteron explosion energies and hence the neutron yields owing to the presence of the higher ionic charge [74-76]. A further advantage is that many of these molecular gases have much larger condensation parameters than $\mathrm{D}_{2}$, eliminating the need for cryogenic cooling to produce large clusters. Another scheme recently demonstrated is the "machining" of a clustered gas jet target using a second laser pulse [77] to disassemble clusters in the lower density entrance wing of the jet, ahead of the arrival of the main heating pulse. By reducing absorption in this wing - known to limit the fusion yield [69] - this has been shown to significantly increase the deposition of laser energy in the high density, central part of the gas jet. A significant increase in both the number of neutrons per shot, and the pulse repetition rate of the neutron source ( $1 \mathrm{kHz}$ is already feasible) is likely to usher in applications of this table-top source, including short pulse neutron imaging, material probing and long term testing of materials under high fluxes of neutrons.

\section{Acknowledgements}

Technical support from P. Ruthven and A. Gregory is gratefully acknowledged. The lasercluster research at Imperial College is supported by the EPSRC. JWGT is supported by an EPSRC Advanced Fellowship.

\section{References}

[1] E.W. Becker, K. Bier, W. Henkes, Z. Phys. 146 (1956) 333.

[2] D. Strickland, G. Morou, Opt. Comm. 56 (1985) 219.

[3] see, e.g. M.D. Perry, G. Morou, Science 264 (1994) 917.

[4] A. McPherson, T.S. Luk, B.D. Thompson, K. Boyer, C.K. Rhodes, Appl. Phys. B 57 (1993) 337.

[5] P. Agostini, G. Petite, Contemp. Phys. 29 (1988) 57.

[6] K. Burnett, V.C. Reed, P.L. Knight, J. Phys. B 26 (1993) 561.

[7] M. Protopapas, C.H. Keitel, P.L. Knight, Rep. Prog. Phys. 60 (1997) 389.

[8] B. Sheehy, L.F. DiMaurio, Ann. Rev. Phys. Chem. 47 (1996) 463.

[9] K. Codling, L.J. Frasinski, Contemp. Phys. 35 (1994) 243.

[10] J. Purnell, E.M. Snyder, S. Wei, A.W. Castleman Jr., Chem. Phys. Lett. 229 (1994) 333.

[11] I. Last, I. Schek, J. Jortner, J. Chem. Phys. 107 (1997) 6685.

[12] see, e.g. P. Gibbon, S. Forster, Plas. Phys. Control Fusion 38 (1996) 769.

[13] L. Rymell, H.M. Hertz, Rev. Sci. Instr. 66 (1995) 4916.

[14] S.P. Gordon, T. Donnelly, A. Sullivan, H. Hamster, R.W. Falcone, Opt. Lett. 19 (1994) 484.

[15] T. Ditmire, T. Donnelly, A.M. Rubenchik, R.W. Falcone, M.D. Perry, Phys. Rev. A 53 (1996) 3379.

[16] O.F. Hagena, W. Obert, J. Chem. Phys. 56 (1972) 1793.

[17] R. Karnbach, M. Joppien, J. Stapelfeldt, J. Wörmer, T. Möller, Rev. Sci. Instr. 64 (1993) 2838.

[18] J. Gspann, in: S. Datz (Ed.), Physics of Electronic and Atomic Collisions, North Holland, Amsterdam, 1982, p. 79.

[19] M. Lewerenz, B. Schilling, J.P. Toennies, Chem. Phys. Lett. 206 (1993) 381.

[20] O.F. Hagena, Rev. Sci. Instr. 63 (1992) 2374.

[21] A.J. Bell, J.M. Mestdagh, J. Berlande, X. Biquard, J. Cuvellier, A. Lallement, P. Meynadier, O. Sublemontier, J.P. Visticot, J. Phys. D. Appl. Phys. 26 (1993) 994.

[22] T. Ditmire, E. Springate, J.W.G. Tisch, Y.L. Shao, M.B. Mason, N. Hay, J.P. Marangos, M.H.R. Hutchinson, Phys. Rev. A 57 (1998) 369.

[23] R.A. Smith, T. Ditmire, J.W.G. Tisch, Rev. Sci. Instr. 69 (1998) 3798.

[24] K. Boyer, B.D. Thompson, A. McPherson, C.K. Rhodes, J. Phys. B: At. Mol. Opt. Phys. 27 (1994) 4373.

[25] W. Brunner, Appl. Phys. B 64 (1997) 443.

[26] C. Rose-Petruck, K.J. Schafer, K.R. Wilson, C.P.J. Barty, Phys. Rev. A 55 (1997) 1182.

[27] I. Last, J. Jortner, Phys. Rev. A 62 (2000) 013201.

[28] T. Ditmire, Phys. Rev. A 57 (1998) R4094.

[29] M. Eloy, R. Azambuja, J.T. Mendonça, R. Bingham, Phys. Scr. T 89 (2001) 60.

[30] J. Liu, R. Li, P. Zhu, Z. Xu, J. Liu, Phys. Rev. A 64 (2001) 033426.

[31] H.M. Milchberg, S.J. McNaught, E. Parra, Phys. Rev. E 64 (2001) 056402.

[32] E. Springate, N. Hay, J.W.G. Tisch, M.B. Mason, T. Ditmire, M.H.R. Hutchinson, J.P. Marangos, Phys. Rev. A 61 (2000) 063201. 
[33] J. Zweiback, T. Ditmire, M.D. Perry, Phys. Rev. A 59 (1999) R3166.

[34] E. Springate, N. Hay, J.W.G. Tisch, M.B. Mason, T. Ditmire, J.P. Marangos, M.H.R. Hutchinson, Phys. Rev. A 61 (2000) 044101.

[35] J. Zweiback, R.A. Smith, T.E. Cowan, G. Hays, K.B. Wharton, V.P. Yanovsky, T. Ditmire, Phys. Rev. Lett. 84 (2000) 2634.

[36] M.V. Ammosov, N.B. Delone, V.P. Krainov, Sov. Phys. JETP 64 (1987) 1191.

[37] W. Lotz, Z. Phys. A 216 (1968) 241.

[38] N.H. Burnett, P.B. Corkum, J. Opt. Soc. Am. B 6 (1989) 1195.

[39] J.D. Jackson, Classical Electrodynamics, John Wiley and Sons, New York, 1975.

[40] V.P. Silin, Sov. Phys. JETP 20 (1965) 1510.

[41] A.F. Haught, D.H. Polk, Phys. Fluids 13 (1970) 2825.

[42] L. Spitzer, Physics of Fully Ionized Gases, Interscience, London, 1962.

[43] Y.L. Shao, T. Ditmire, J.W.G. Tisch, E. Springate, J.P. Marangos, M.H.R. Hutchinson, Phys. Rev. Lett. 77 (1996) 3343.

[44] M. Lezius, S. Dobosz, D. Normand, M. Schmidt, J. Phys. B: At. Mol. Opt. Phys. 30 (1997) L251.

[45] J. Purnell, E.M. Snyder, S. Wei, A.W. Castleman Jr., Chem. Phys. Lett. 229 (1994) 333.

[46] E.M. Snyder, S. Wei, J. Purnell, S.A. Buzza, A.W. Castleman Jr., Chem. Phys. Lett. 248 (1996) 1.

[47] T. Ditmire, J.W.G. Tisch, E. Springate, M.B. Mason, N. Hay, R.A. Smith, J.P. Marangos, M.H.R. Hutchinson, Nature 386 (1997) 54.

[48] T. Ditmire, R.A. Smith, J.W.G. Tisch, M.H.R. Hutchinson, Phys. Rev. Lett. 78 (1997) 3121.

[49] M. Lezius, S. Dobosz, D. Normand, M. Schmidt, Phys. Rev. Lett. 80 (1998) 261.

[50] D.J. Fraser, M.H.R. Hutchinson, J. Mod. Opt. 43 (1996) 1055.

[51] S. Augst, D. Strickland, D.D. Meyerhofer, S.L. Chin, J.H. Eberly, Phys. Rev. Lett. 63 (1989) 2212.

[52] C. Cornaggia, M. Schmidt, D. Normand, J. Phys. B: At. Mol. Opt. Phys. 27 (1994) L123.

[53] S. Augst, D.D. Meyerhofer, D. Strickland, S.L. Chin, J. Opt. Soc. B 8 (1991) 858.

[54] E. Springate, N. Hay, J.W.G. Tisch, M.B. Mason, T. Ditmire, M.H.R. Hutchinson, J.P. Marangos, Phys. Rev. A 61 (2000) 063201.

[55] K.J. Mendham, N. Hay, M.B. Mason, J.W.G. Tisch, J.P. Marangos, Phys. Rev. A 64 (2001) 055201.

[56] E. Parra, I. Alexeev, J. Fan, K.Y. Kim, S.J. McNaught, H.M. Milchberg, Phys. Rev. E 62 (2000) R5931.

[57] K.J. Mendham, Energetic cluster explosions in intense, femtosecond laser fields, Ph.D. thesis, University of London, 2002.

[58] T. Ditmire, R.A. Smith, J.W.G. Tisch, M.H.R. Hutchinson, Phys. Rev. Lett. 78 (1997) 3121.
[59] C.W. Gwyn, R. Stulen, D. Sweeney, D. Attwood, J. Vac. Sci. Technol. B 16 (1998) 3142.

[60] G.C. Junkel-Vives, J. Abdallah, T. Auguste, P. D’Oliveira, S. Hulin, P. Monot, S. Dobosz, A.Y. Faenov, A.I. Magunov, T.A. Pikuz, et al., Phys. Rev. E 65 (2002) 036410.

[61] J. Larsson, A. Sjogren, Rev. Sci. Instr. 70 (1999) 2253.

[62] S. Dobosz, M. Lezius, M. Schmidt, P. Meynadier, M. Perdrix, D. Normand, J.P. Rozet, D. Vernhet, Phys. Rev. A 56 (1997) R2526.

[63] S. Ter-Avetisyan, M. Schnürer, H. Stiel, U. Vogt, W. Radloff, W. Karpov, W. Sandner, P.V. Nickles, Phys. Rev. E 64 (2001) 036404.

[64] M. Schnürer, S. Ter-Avetisyan, H. Stiel, U. Vogt, W. Radloff, M. Kalashnikov, W. Sandner, P.V. Nickles, Eur. Phys. J. D 14 (2001) 331.

[65] M. Mori, T. Shiraishi, E. Takahashi, H. Suzuki, L.B. Sharma, E. Miura, K. Kondo, J. Appl. Phys. 90 (2001) 3595 .

[66] K. Kondo, A.B. Borisov, C. Jordan, A. McPherson, W.A. Schroeder, K. Boyer, C.K. Rhodes, J. Phys. B 30 (1997) 2707.

[67] T. Ditmire, P.K. Patel, R.A. Smith, J.S. Wark, S.J. Rose, D. Milathianaki, R.S. Marjoribanks, M.H.R. Hutchinson, J. Phys. B 31 (1998) 2825.

[68] T. Ditmire, J. Zweiback, V.P. Yanovsky, T.E. Cowan, G. Hays, K.B. Wharton, Nature (London) 398 (1999) 489.

[69] J. Zweiback, T.E. Cowan, J.H. Hartley, R. Howell, K.B. Wharton, J.K. Crane, V.P. Yanovsky, G. Hays, R.A. Smith, T. Ditmire, Phys. Plasma 9 (2002) 3108.

[70] P.B. Parks, T.E. Cowan, R.B. Stephens, E.M. Campbell, Phys. Rev. A 63 (2001) 063203.

[71] Y. Kishimoto, T. Masaki, T. Tajima, Phys. Plasma 9 (2002) 589.

[72] K.J. Mendham, J.W.G. Tisch, M.B. Mason, N. Hay, R.A. Smith, J.P. Marangos, J. Phys. B 35 (2002) 663.

[73] J. Zweiback, T.E. Cowan, R.A. Smith, J.H. Hartley, R. Howell, C.A. Steinke, G. Hays, K.B. Wharton, J.K. Crane, T. Ditmire, Phys. Rev. Lett. 85 (2000) 3640.

[74] J.W.G. Tisch, N. Hay, E. Springate, E.T. Gumbrell, M.H.R. Hutchinson, J.P. Marangos, Phys. Rev. A 60 (1999) 3076.

[75] I. Last, J. Jortner, Phys. Rev. Lett. 87 (2001) 033401.

[76] G. Grillon, Ph. Balcou, J.P. Chambaret, D. Hulin, J. Martino, S. Moustaizis, L. Notebaert, M. Pittman, Th. Pussieux, A. Rousse, et al., Phys. Rev. Lett. 89 (2002) 065005.

[77] D.R. Symes, A.J. Comley, J.W.G. Tisch, R.A. Smith, Appl. Phys. Lett. 80 (2002) 4112.

[78] F. Blasco, T. Caillaud, F. Dorchies, C. Stenz, J. Stevefelt, A.S. Boldarev, V.A. Gasilov, Nucl. Instr. and Meth. B, these Proceedings. doi:10.1016/S0168-583X(02)01983-3.

[79] L. Adoui, O. Gobert, P. Indelicato, E. Lamour, P. Meynadier, D. Normand, M. Perdrix, C. Prigent, J.-P. Rozet, D. Vernhet, Nucl. Instr. and Meth. B, these Proceedings. doi:10.1016/S0168-583X(03)00594-9. 